\title{
Pressure-induced collapse of the spin-orbital Mott state in the hyperhoneycomb iridate $6-\mathrm{Li}_{2} \mid \mathrm{IO}_{3}$
}

\author{
T. Takayama, A. Krajewska, A. S. Gibbs, A. N. Yaresko, \\ H. Ishii, H. Yamaoka, K. Ishii, N. Hiraoka, N. P. Funnell, \\ C. L. Bull and H. Takagi
}

\section{Published version information}

Citation: T Takayama et al. "Pressure-induced collapse of the spin-orbital Mott state in the hyperhoneycomb iridate $\beta$-Li2IrO3." Physical Review B, vol. 99, no. 12 (2019): 125127.

DOI: $10.1103 /$ PhysRevB.99.125127

This version is made available in accordance with publisher policies. Please cite only the published version using the reference above. This is the citation assigned by the publisher at the time of issuing the APV. Please check the publisher's website for any updates. 


\title{
Pressure-induced collapse of the spin-orbital Mott state in the hyperhoneycomb iridate $\beta$ - $\mathrm{Li}_{2} \mathrm{IrO}_{3}$
}

\author{
T. Takayama, ${ }^{1,2}$ A. Krajewska,,${ }^{1,2}$ A. S. Gibbs, ${ }^{3}$ A. N. Yaresko, ${ }^{1}$ H. Ishii, ${ }^{4}$ H. Yamaoka, ${ }^{5}$ K. Ishii, ${ }^{6}$ \\ N. Hiraoka, ${ }^{4}$ N. P. Funnell, ${ }^{3}$ C. L. Bull, ${ }^{3}$ and H. Takagi $1,2,7$ \\ ${ }^{1}$ Max Planck Institute for Solid State Research, Heisenbergstrasse 1, 70569 Stuttgart, Germany \\ ${ }^{2}$ Institute for Functional Matter and Quantum Technologies, University of Stuttgart, Pfaffenwaldring 57, 70550 Stuttgart, Germany \\ ${ }^{3}$ ISIS Neutron and Muon Source, STFC Rutherford Appleton Laboratory, Chilton, Didcot, Oxon OX11 OQX, United Kingdom \\ ${ }^{4}$ National Synchrotron Radiation Research Center, Hsinchu 30076, Taiwan \\ ${ }^{5}$ RIKEN SPring-8 Center, Sayo, Hyogo 679-5148, Japan \\ ${ }^{6}$ Synchrotron Radiation Research Center, National Institutes for Quantum and Radiological Science and Technology, \\ Sayo, Hyogo 679-5148, Japan \\ ${ }^{7}$ Department of Physics, University of Tokyo, 7-3-1 Hongo, Tokyo 113-0033, Japan
}

(Received 16 August 2018; revised manuscript received 4 March 2019; published 18 March 2019)

\begin{abstract}
Hyperhoneycomb iridate $\beta$ - $\mathrm{Li}_{2} \mathrm{IrO}_{3}$ is a three-dimensional analog of two-dimensional honeycomb iridates, such as $\alpha-\mathrm{Li}_{2} \mathrm{IrO}_{3}$, which recently appeared as another playground for the physics of Kitaev-type spin liquid. $\beta$ - $\mathrm{Li}_{2} \mathrm{IrO}_{3}$ shows a noncollinear spiral ordering of spin-orbital-entangled $J_{\text {eff }}=1 / 2$ moments at low temperatures below $38 \mathrm{~K}$, which is known to be suppressed under a pressure of $\sim 2 \mathrm{GPa}$. In addition, a structural transition is observed at $P_{\mathrm{S}} \sim 4 \mathrm{GPa}$ at room temperature. Using the neutron powder diffraction technique, the crystal structure in the high-pressure phase of $\beta-\mathrm{Li}_{2} \mathrm{IrO}_{3}$ above $P_{\mathrm{S}}$ was refined, which indicates the formation of $\mathrm{Ir}_{2}$ dimers on the zigzag chains, with an Ir-Ir distance of $\sim 2.66 \AA$, even shorter than that of metallic Ir. We argue that the strong dimerization stabilizes the bonding molecular-orbital state comprising the two local $d_{z x}$ orbitals in the Ir- $\mathrm{O}_{2}$-Ir bond plane, which conflicts with the equal superposition of $d_{x y}, d_{y z}$, and $d_{z x}$ orbitals in the $J_{\text {eff }}=1 / 2$ wave function produced by strong spin-orbit coupling. The results of resonant inelastic x-ray scattering measurements and the electronic structure calculations are fully consistent with the collapse of the $J_{\text {eff }}=1 / 2$ state. The competition between the spin-orbital-entangled $J_{\text {eff }}=1 / 2$ state and molecular-orbital formation is most likely universal in honeycomb-based Kitaev materials.
\end{abstract}

DOI: 10.1103/PhysRevB.99.125127

\section{INTRODUCTION}

The Kitaev model, with $S=1 / 2$ spins on a honeycomb lattice connected by a bond-dependent Ising coupling, has been attracting considerable interest as it provides an exactly solvable quantum-spin-liquid (QSL) ground state consisting of the two kinds of Majorana fermions [1]. The materialization of the Kitaev QSL has been pursued extensively in honeycomb-based spin-orbital Mott insulators with heavy transition-metal ions with $d^{5}\left(t_{2 g}^{5}\right)$ configuration, such as $\mathrm{Ir}^{4+}$ and $\mathrm{Ru}^{3+}[2,3]$. In these spin-orbital compounds, the heavy $d^{5}$ transition-metal ions are octahedrally coordinated with anions, and the octahedra form a honeycomb network by sharing their edges. The strong spin-orbit coupling $\lambda_{\mathrm{SO}} \sim 0.5 \mathrm{eV}$ for $\mathrm{Ir}^{4+}$ and $0.1 \mathrm{eV}$ for $\mathrm{Ru}^{3+}$ splits the degenerate $t_{2 g}^{5}$ into the half-filled $J_{\text {eff }}=1 / 2$ doublet and the completely filled $J_{\text {eff }}=3 / 2$ quartet [4]. The magnetism of the candidate compounds therefore originates from $J_{\text {eff }}=1 / 2$ pseudospins. The superexchange coupling between two adjacent $J_{\text {eff }}=1 / 2$ moments was theoretically proposed to be a bond-dependent ferromagnetic Ising interaction as in the Kitaev model [5].

The layered honeycomb iridates $\mathrm{Na}_{2} \mathrm{IrO}_{3}$ and $\alpha-\mathrm{Li}_{2} \mathrm{IrO}_{3}$ emerged as the first generation of candidate materials for the Kitaev QSL [6,7]. Their ground state, however, turned out not to be a QSL. They were found to show a magnetic transition to a zigzag-type antiferromagnetic phase $[8,9]$ and to a noncoplanar spiral phase [10], respectively, at a low temperature.
$\alpha-\mathrm{RuCl}_{3}$ was subsequently proposed as the first noniridiumbased candidate but again was found to show a zigzagtype antiferromagnetic ordering as in $\mathrm{Na}_{2} \mathrm{IrO}_{3}$ [11-14]. In parallel with these, three-dimensional (3D) analogs of the two-dimensional (2D) honeycomb $\alpha-\mathrm{Li}_{2} \mathrm{IrO}_{3}, \beta-\mathrm{Li}_{2} \mathrm{IrO}_{3}$ and $\gamma-\mathrm{Li}_{2} \mathrm{IrO}_{3}$, were discovered as another platform for Kitaev magnetism $[15,16]$. These 3D honeycomb compounds also show a clear magnetic transition into a complex spiral phase $[17,18]$ similar to that of $\alpha-\mathrm{Li}_{2} \mathrm{IrO}_{3}$, although a closer proximity to the Kitaev spin liquid than their 2D analogs is suggested $[19,20]$. For example, the strong magnetic anisotropy $[16,21]$ and the overall ferromagnetic interactions with positive Curie-Weiss temperature $\left(\theta_{\mathrm{CW}} \sim+40 \mathrm{~K}\right.$ for polycrystalline $\beta$ - $\mathrm{Li}_{2} \mathrm{IrO}_{3}$ [15]) suggest the predominance of bonddependent Kitaev coupling. The presence of magnetic interactions other than the Kitaev coupling, such as a direct Heisenberg exchange and off-diagonal coupling, has been discussed to stabilize the long-range magnetic ordering instead of a QSL state $[2,3]$.

In the 2D honeycomb iridates, a chemical substitution of the interlayer $\mathrm{Li}$ ions was attempted to tune the magnetic interactions through a local lattice distortion and to bring the ground state closer to the QSL [22-24]. With such an approach, $\mathrm{H}_{3} \mathrm{LiIr}_{2} \mathrm{O}_{6}$ was very recently found to host a QSL ground state $[25,26]$, while the relevance to Kitaev physics remains to be identified. Control of the ground states using 
magnetic field was also attempted. By applying a magnetic field of $\mu_{0} H_{c} \sim 8 \mathrm{~T}$ parallel to the honeycomb planes in $\alpha-\mathrm{RuCl}_{3}$, the disappearance of the magnetic ordering was observed, and the emergence of a QSL-like phase was pointed out, which has been the subject of intensive studies [12,27-31]. The analogous field-induced suppression of spiral magnetic order was observed in 3D honeycomb iridates $\beta$ and $\gamma-\mathrm{Li}_{2} \mathrm{IrO}_{3}$ by applying magnetic field along the $b$ axis $[21,32]$, potentially giving rise to a similar QSL-like ground state.

Another promising approach to control the magnetic ground state may be the application of pressure. The emergence of a QSL state under pressure was theoretically proposed in honeycomb-based iridates [33-35]. Indeed, the suppression of long-range magnetic order under high pressure was reported in 3D honeycomb iridates $\beta-\mathrm{Li}_{2} \mathrm{IrO}_{3}$ and $\gamma$ - $\mathrm{Li}_{2} \mathrm{IrO}_{3}$ at low temperatures in $\mathrm{x}$-ray magnetic circular dichroism (XMCD) [15,36], resonant magnetic $x$-ray scattering [37], and muon spin rotation measurements [38] by applying a pressure of $1-2 \mathrm{GPa}$, while no appreciable structural distortion is seen in the pressure range [37]. $\mathrm{In} \beta-\mathrm{Li}_{2} \mathrm{IrO}_{3}$, a firstorder pressure-induced structural transition to a monoclinic structure at critical pressure $P_{\mathrm{S}} \sim 4 \mathrm{GPa}$ was discovered by single-crystal $x$-ray diffraction at room temperature [36]. The high-pressure monoclinic structure contains a reduced Ir-Ir bond length in the zigzag chain, and possible dimer formation was invoked. However, the impact of this structural phase transition on the electronic structure remains to be clarified.

In this paper, we confirmed by neutron diffraction that the structural transition at $P_{\mathrm{S}} \sim 4 \mathrm{GPa}$ in $\beta-\mathrm{Li}_{2} \mathrm{IrO}_{3}$ is accompanied by the formation of $\operatorname{Ir}_{2}$ dimers on the one-dimensional zigzag chains. The resonant inelastic $\mathrm{x}$-ray scattering measurement indicates a breakdown of the $J_{\text {eff }}=1 / 2$ description in the high-pressure dimerized phase. The electronic structure calculation indicates that the dimerization gives rise to the formation of local molecular orbitals and hence the collapse of the $J_{\text {eff }}=1 / 2$ state. The formation of dimers may give a clue to the origin of the putative QSL behavior appearing under pressure at low temperatures and points to a potentially universal competition between the spin-orbital entanglement and molecular-orbital formation of Ir $d$ electrons in the honeycomb-based iridates.

\section{EXPERIMENT}

Neutron diffraction measurements on a powder sample of $\beta-\mathrm{Li}_{2} \mathrm{IrO}_{3}$ were performed under pressure to unveil the detailed crystal structure of the high-pressure phase. The use of neutron diffraction allowed the reliable and precise refinement of positions not only for the heavy Ir atoms but also for light $\mathrm{Li}$ and $\mathrm{O}$ atoms to which neutrons are intrinsically much more sensitive than $\mathrm{x}$ rays. The measurements were conducted at the PEARL beamline of the ISIS Neutron and Muon Source [39]. Pressure was applied by a Paris-Edinburgh press up to $5.2 \mathrm{GPa}$ [40]. The anvils were single-toroidal zirconia-toughened alumina, and an encapsulated TiZr gasket was used [41]. In order to minimize the neutron absorption by ${ }^{191} \mathrm{Ir}$, we prepared an isotope-enriched powder sample of $\beta-{ }^{7} \mathrm{Li}_{2}{ }^{193} \mathrm{IrO}_{3}$ [42]. The powder sample was synthesized by a conventional solid-state reaction using powder of ${ }^{7} \mathrm{Li}_{2} \mathrm{CO}_{3}$ and metallic ${ }^{193}$ Ir. A deuterium-substituted methanol-ethanol mixture (4:1 by volume) was used as a hydrostatic pressure medium. The applied pressure was calibrated from the lattice constant of $\mathrm{NaCl}$ powder added as a pressure marker. All of the measurements were conducted at room temperature. The Rietveld refinement of diffraction patterns was performed by assuming the presence of five phases, $\beta-\mathrm{Li}_{2} \mathrm{IrO}_{3}$, metallic Ir as an impurity $\left(\sim 8\right.$ wt $\%$ of $\left.\beta-\mathrm{Li}_{2} \mathrm{IrO}_{3}\right), \mathrm{NaCl}$, and anvil materials $\left(\mathrm{ZrO}_{2}\right.$ and $\left.\mathrm{Al}_{2} \mathrm{O}_{3}\right)$, using the GSAS program [43].

To investigate the electronic structure under pressure, we performed resonant inelastic x-ray scattering (RIXS) measurements with Ir $L_{3}$ edge on $\beta-\mathrm{Li}_{2} \mathrm{IrO}_{3}$ at $\mathrm{BL} 12 \mathrm{XU}$ of SPring-8. A diamond anvil cell (DAC) was used for the application of pressure. A small single crystal of 50- $\mu \mathrm{m}$ size, grown by a flux method using $\mathrm{LiCl}$ [15], was loaded in a DAC with Fluorinert (1:1 mixture of FC-70 and FC-77 by volume) as a pressure medium [44]. Pressure was evaluated by the fluorescence spectra of a ruby ball loaded together with the sample. A gasket made of beryllium was used so that the incident and scattered $x$ rays go through the gasket with minimum attenuation. The energy of incident $x$ rays was tuned to 11.215 $\mathrm{keV}$, which corresponds to Ir $2 p_{3 / 2} \rightarrow 5 d\left(t_{2 g}\right)$ excitation.

The incident $\mathrm{x}$-ray beam was monochromated by a $\mathrm{Si}(111)$ double-crystal monochromator and further by a four-bounce $\mathrm{Si}(440)$ high-resolution monochromator and was focused by using a Kirkpatrick-Baez mirror. The scattering angle $(2 \theta)$ was kept at $90^{\circ}$, and $\pi$-polarized incident $\mathrm{x}$ rays were used to minimize the elastic scattering. The scattered $\mathrm{x}$ rays were analyzed by a diced and spherically bent $\mathrm{Si}(844)$ analyzer. The total energy resolution, estimated from the full width at half maximum of the elastic line, was about $100 \mathrm{meV}$. The alignment of the sample position was performed by monitoring the intensity of the Ir $L \alpha_{\mathrm{I}}$ emission line ( $\left.3 d \rightarrow 2 p_{3 / 2}\right)$ by using a Ge(733) spherically bent analyzer. The sample alignment was repeated after the pressure was changed. No $\boldsymbol{q}$-resolved measurements were performed, and the spectra were collected at an unknown $\boldsymbol{q}$ position of the single crystal. Since the $d-d$ excitations of honeycomb iridates were shown to display no appreciable $\boldsymbol{q}$ dependence [45], the obtained spectra are supposed to represent essential features of $d$ - $d$ excitations. For reference, we also collected the RIXS spectrum of polycrystalline $\beta-\mathrm{Li}_{2} \mathrm{IrO}_{3}$ at ambient pressure without using a DAC. All data were collected at room temperature.

The electronic structure calculations were performed using the crystal structures of the ambient- and high-pressure phases, which were refined from the neutron diffraction data. The calculations were carried out based on the local-density approximation using a fully relativistic linear muffin-tin orbital (LMTO) method implemented in the PY LMTO code [46]. Spin-orbit coupling was taken into account by solving the four-component Dirac equation inside an atomic sphere. This allows us to obtain densities of states resolved by the total angular momentum $J$.

\section{RESULTS}

\section{A. Structural transition under pressure}

The result of the structure refinement at ambient pressure from neutron diffraction data is shown in Fig. S1(a) and 


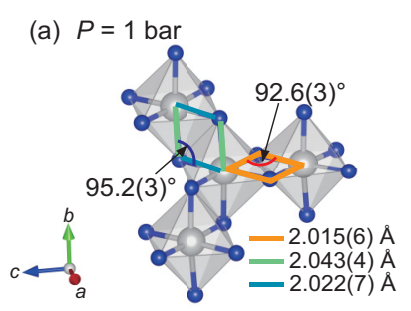

(b) Fddd

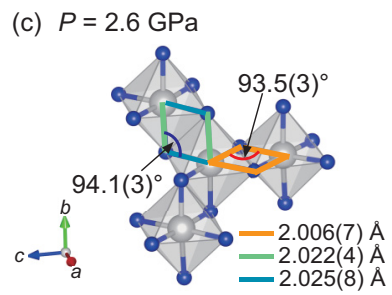

(e) $P=4.4 \mathrm{GPa}$

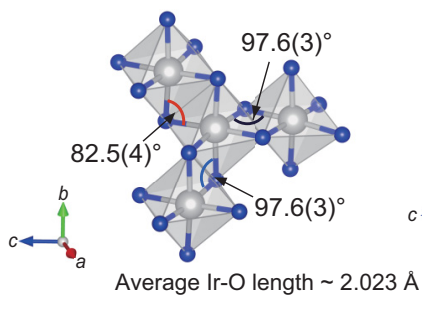

(f) $\mathrm{C} 2 / \mathrm{c}$

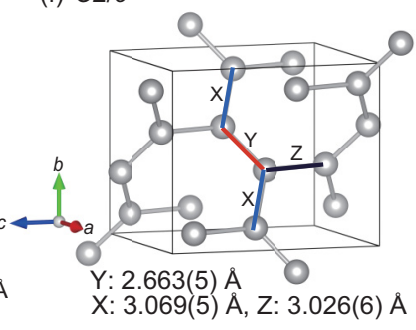

FIG. 1. Crystal structure of $\beta-\mathrm{Li}_{2} \mathrm{IrO}_{3}$. (a) and (c) Local structure around an $\mathrm{IrO}_{6}$ octahedron at ambient pressure and 2.6(1) GPa, respectively. (b) and (d) Hyperhoneycomb network of Ir atoms. X, $\mathrm{Y}$, and $\mathrm{Z}$ denote the three types of Ir-Ir bonds. (e) Local structure around an $\mathrm{IrO}_{6}$ octahedron in the high-pressure phase at 4.4(1) GPa. (f) Ir sublattice in the high-pressure phase. The dimerized bond is shown in red. The crystal structures are illustrated using VESTA software [47].

Table S1 in the Supplemental Material [44], which agrees very well with that obtained by single-crystal $\mathrm{x}$-ray diffraction [15]. $\beta$ - $\mathrm{Li}_{2} \mathrm{IrO}_{3}$ crystallizes in a distorted version of ordered rocksalt-type structure. Each $\mathrm{IrO}_{6}$ octahedron shares its edges with the three neighboring $\mathrm{IrO}_{6}$ octahedra as in $\alpha-\mathrm{Li}_{2} \mathrm{IrO}_{3}$ [15]. The local configuration of bonds around an $\mathrm{IrO}_{6}$ octahedron is illustrated in Fig. 1(a). All of the $\mathrm{IrO}_{6}$ octahedra are crystallographically equivalent and form a three-dimensional network via the three almost $120^{\circ}$ bonds, termed a hyperhoneycomb lattice. The sublattice of Ir atoms at room temperature and at ambient pressure is depicted in Fig. 1(b). The hyperhoneycomb lattice can be viewed as an assembly of Ir zigzag chains, running along the $\boldsymbol{a}+\boldsymbol{b}$ and the $\boldsymbol{a}-\boldsymbol{b}$ directions alternately. The zigzag chains are bridged by the Ir-Ir bonds along the $c$ axis. The three $120^{\circ}$ Ir-Ir bonds can be labeled as $\mathrm{X}, \mathrm{Y}$, and $\mathrm{Z}$ bonds; the $\mathrm{Z}$ bond is the bridging bond along the $c$ axis, and the $\mathrm{X}$ and $\mathrm{Y}$ bonds form the zigzag chains. In the orthorhombic structure at ambient pressure (space group $F d d d$ ), the $\mathrm{X}$ and $\mathrm{Y}$ bonds are symmetry equivalent. The $\mathrm{X}$ and the $\mathrm{Y}$ bonds are appreciably longer than the $\mathrm{Z}$ bond by $3 \%$ at ambient pressure.

With the application of pressure, the orthorhombic unit cell displays an anisotropic contraction as reported in Ref. [36] (see Supplemental Material [44]). The $b$-axis lattice constant shows a stronger pressure dependence than those of the $a$ axis
TABLE I. Refined structural parameters of $\beta-\mathrm{Li}_{2} \mathrm{IrO}_{3}$ in the highpressure phase at 4.4(1) GPa. The space group is $C 2 / c$ (No. 15), and $Z=8$. The lattice parameters are $a=5.8390(4) \AA, b=8.1297(5)$ $\AA, c=9.2240(6) \AA$, and $\beta=106.658(4)^{\circ} . g$ and $U_{\text {iso }}$ denote site occupancy and the isotropic displacement parameter, respectively. $U_{\text {iso }}$ was constrained to be equal across sites containing the same element during the refinement. The refinement indices are $R_{p}=$ $2.69 \%, R_{w p}=2.27 \%$, and $\chi^{2}=2.472$. The Rietveld fits to the data are available in the Supplemental Material [44].

\begin{tabular}{lcccccc}
\hline \hline Atom & Site & $g$ & \multicolumn{1}{c}{$x$} & \multicolumn{1}{c}{$y$} & \multicolumn{1}{c}{$z$} & $U_{\text {iso }}\left(\AA^{2}\right)$ \\
\hline Li1 & $8 f$ & 1 & $0.261(4)$ & $0.6439(18)$ & $0.2437(22)$ & $0.0118(15)$ \\
Li2 & $8 f$ & 1 & $0.9345(28)$ & $0.6255(22)$ & $0.6007(15)$ & $0.0118(15)$ \\
Ir1 & $8 f$ & 1 & $0.4241(6)$ & $0.3857(5)$ & $0.0788(5)$ & $0.0058(4)$ \\
O1 & $8 f$ & 1 & $0.7262(10)$ & $0.3894(8)$ & $0.2516(7)$ & $0.0058(3)$ \\
O2 & $8 f$ & 1 & $0.9025(12)$ & $0.3614(6)$ & $0.5816(8)$ & $0.0058(3)$ \\
O3 & $8 f$ & 1 & $0.4067(12)$ & $0.3666(6)$ & $0.5835(8)$ & $0.0058(3)$ \\
\hline \hline
\end{tabular}

and the $c$ axis, which results from the rapid contraction of $\mathrm{X}$ and $\mathrm{Y}$ bonds. By comparing the bond lengths at ambient pressure [Fig. 1(b)] and at a pressure of 2.6(1) GPa [Fig. 1(d)], the $\mathrm{X}$ - and Y-bond lengths decrease by $1.3 \%$, whereas that of the $\mathrm{Z}$ bond actually increases by $0.5 \%$. As a result, the $\mathrm{X}$ and Y-bond lengths and Z-bond length become much closer at $2.6 \mathrm{GPa}$ than at ambient pressure. The Ir-O bond lengths do not show any appreciable change from ambient pressure to $2.6 \mathrm{GPa}$, meaning that the change in the Ir-Ir bond lengths is controlled by the Ir-O-Ir angle. In agreement with this, the Ir-O-Ir angles for $\mathrm{X}$ and $\mathrm{Y}$ bonds and the $\mathrm{Z}$ bond became much closer [Figs. 1(c) and 1(d)].

With a further increase of pressure, a structural transition from the low-pressure orthorhombic $(F d d d)$ to the highpressure monoclinic $(C 2 / c)$ structure takes place at around 3.7 GPa, consistent with Ref. [36]. The result of structural refinement for the high-pressure phase at 4.4(1) GPa is listed in Table I. The hyperhoneycomb network made of edgeshared $\mathrm{IrO}_{6}$ octahedra is maintained as shown in Figs. 1(e) and 1(f) (see the Supplemental Material for the details of local structure). The average length of $\mathrm{X}$ and $\mathrm{Y}$ bonds along the zigzag chains decreases further to $\sim 2.87 \AA$, and the bridging Z-bond length increases to $3.026(6) \AA$. This appears to be an extension of the anisotropic pressure dependence between $\mathrm{X}(=\mathrm{Y})$ and $\mathrm{Z}$ in the low-pressure phase. A modulation of the Ir-Ir bond length in the zigzag chains, however, makes the high-pressure phase distinct from the low-pressure phase. As seen in Fig. 1(f), the $\mathrm{X}$ bond and $\mathrm{Y}$ bond are no longer equivalent at 4.4 GPa. While the $\mathrm{X}$-bond length is as long as 3.069(5) $\AA$, close to or even longer than the increased Z-bond length, the Y-bond length becomes as short as 2.663(5) $\AA$, which gives rise to an alternating arrangement of the short $\mathrm{Y}$ bonds and the long $\mathrm{X}$ bonds along the zigzag chains. In fact, at a distance of $2.66 \AA$, the Ir-Ir Y bond is even shorter than that seen in metallic $\operatorname{Ir}(\sim 2.71 \AA)$, indicating the formation of an $\mathrm{Ir}_{2}$ dimer molecule in the zigzag chains [48].

\section{B. Collapse of the $J_{\text {eff }}=1 / 2$ state in the high-pressure dimerized phase}

RIXS measurements unveil the drastic reconstruction of the electronic structure associated with this dimerization. The 

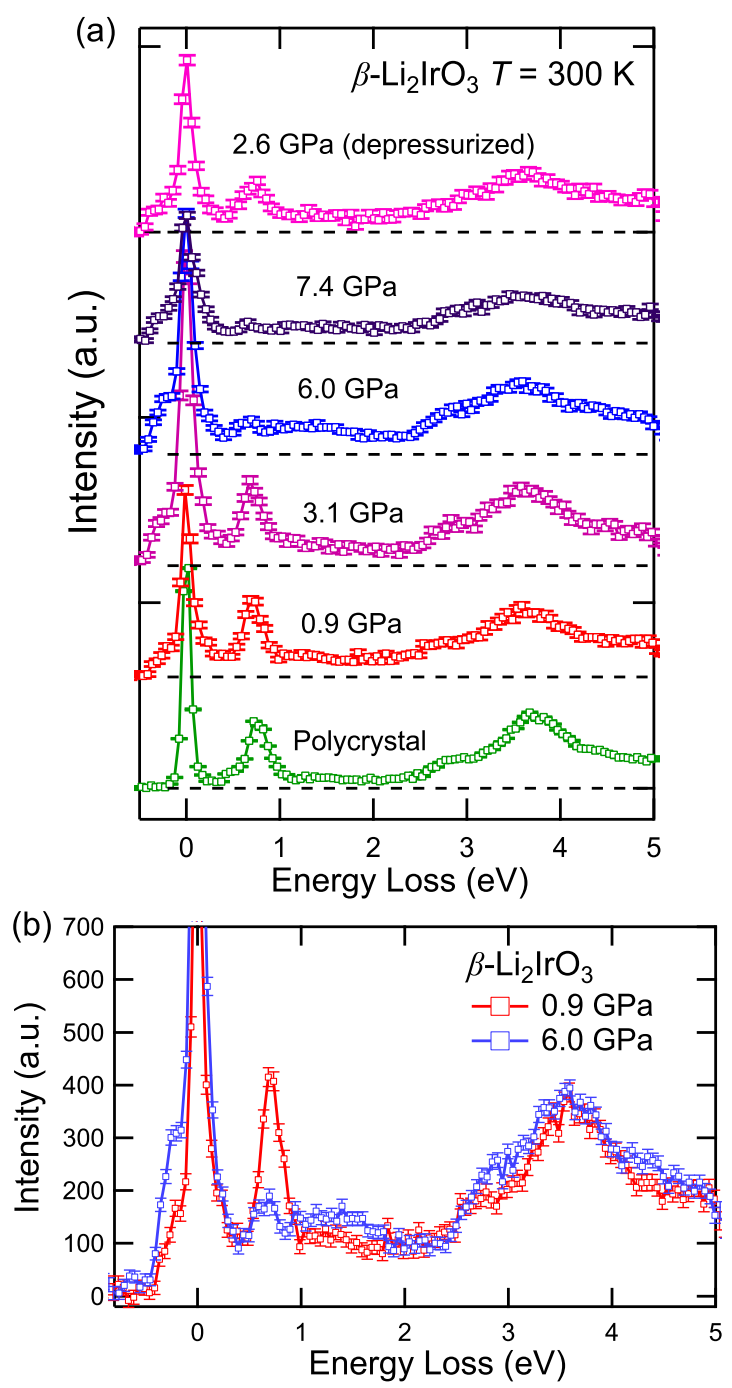

FIG. 2. RIXS spectra of $\beta-\mathrm{Li}_{2} \mathrm{IrO}_{3}$ under pressure recorded at room temperature. The data from the polycrystalline sample (bottom) were collected at ambient pressure, and the data at 2.6(1) GPa (top) were collected after the sample was depressurized from 7.4(1) GPa. The spectra are normalized by the high-energy tail above $5 \mathrm{eV}$ and shown with arbitrary offsets. The horizontal dashed lines represent the guide baselines, obtained by subtracting a constant background from each spectrum. (b) The comparison of RIXS spectra between the ambient and high-pressure phases above $P_{\mathrm{S}}$. The spectra are normalized by the high-energy tails above $5 \mathrm{eV}$ to highlight the change by pressure. The low-energy shoulder of the elastic line in the 6.0-GPa spectrum is likely attributed to the scattering from the Be gasket or pressure medium.

Ir $L_{3}$-edge RIXS spectrum at ambient pressure, measured on a polycrystalline pellet, is displayed at the bottom of Fig. 2 . In addition to the elastic scattering peak at $0 \mathrm{eV}$, there are two pronounced features: a sharp peak at around $0.7 \mathrm{eV}$ and a broad peak centered at around $3.5 \mathrm{eV}$. The latter represents the excitations from Ir $5 d t_{2 g}$ to $e_{g}$ manifolds. The peak at $\sim 0.7 \mathrm{eV}$ can be assigned to the local excitation between the filled $J_{\text {eff }}=3 / 2$ and the half-filled $J_{\text {eff }}=1 / 2$ state, which has been observed in a number of $d^{5}$ iridium oxides [45,49-51]. Together with the previous XMCD result [15] and the

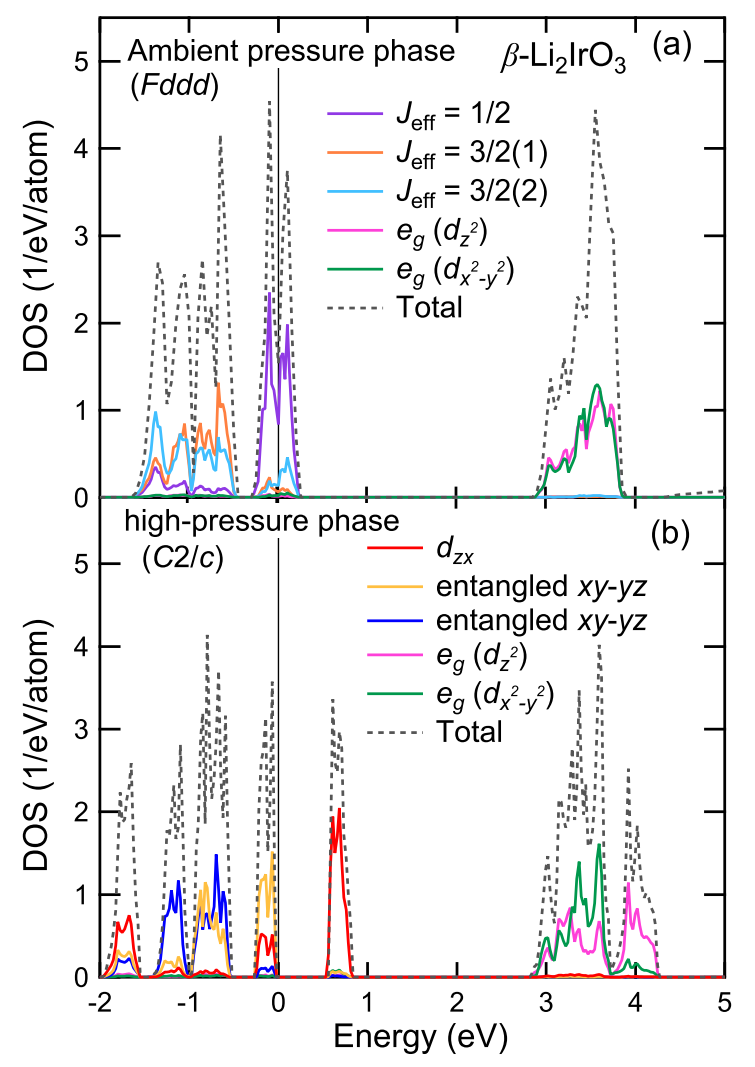

FIG. 3. Calculated density of states (DOS) for Ir $5 d$ states. (a) DOS for the ambient pressure phase $(F d d d)$. The Ir $t_{2 g}$ orbitals are resolved into $J_{\text {eff }}=1 / 2$ and $3 / 2$ states. The two $J_{\text {eff }}=3 / 2$ states, (1) and (2), are primarily composed of $J_{\text {eff }}^{z}=1 / 2$ and $J_{\text {eff }}^{z}=3 / 2$ characters, respectively. (b) DOS for the high-pressure phase $(C 2 / c)$. The $d_{z x}$ orbital is directed along the $\operatorname{Ir}_{2}$ dimer bond. The other $t_{2 g}$ orbitals, $d_{x y}$ and $d_{y z}$, are entangled by spin-orbit coupling and denoted as "entangled $x y-y z . "$ The total DOS includes the contributions from oxygen $2 p$ states.

electronic structure calculations (Refs. [19,20] and Fig. 3(a)), this supports the dominant $J_{\text {eff }}=1 / 2$ character of $t_{2 g}$ holes in $\beta-\mathrm{Li}_{2} \mathrm{IrO}_{3}$ at ambient pressure.

RIXS spectra under pressure were collected with a single crystal loaded in a DAC. At a low pressure of $0.9(1) \mathrm{GPa}$, the spectrum for the single crystal agrees well with that of the polycrystalline sample at ambient pressure. This supports the idea that the $d$ - $d$ excitations show only a small $\boldsymbol{q}$ dependence in $\beta-\mathrm{Li}_{2} \mathrm{IrO}_{3}$. With increasing pressure up to 3.1(1) GPa, the $0.7 \mathrm{eV}$ peak remains at the same energy, indicating that the $J_{\text {eff }}=1 / 2$ state is robust in this pressure range (see Supplemental Material, Fig. S5, for the details). This is consistent with the absence of a structural phase transition in this pressure range [Figs. 1(c) and 1(d)] and contrasts with the 2D analog of $\alpha-\mathrm{Li}_{2} \mathrm{IrO}_{3}$, where the breakdown of the $J_{\text {eff }}=1 / 2$ picture at a small pressure below $1 \mathrm{GPa}$ has been discussed [52]. A pressure-induced change in electronic structure, in the pressure range below $2 \mathrm{GPa}$, was also inferred in $\beta-\mathrm{Li}_{2} \mathrm{IrO}_{3}$ at low temperatures by the $\mathrm{x}$-ray absorption spectra where the disappearance of magnetic-field-induced moment and the pronounced suppression of the branching ratio were found [36]. We note that these were observed at low temperatures 
below $10 \mathrm{~K}$, not at room temperature. The critical pressure of the structural transition at $10 \mathrm{~K}$ is not known yet. More detailed investigation of RIXS in the temperature-pressure region might give us a hint to draw a coherent picture of pressure-induced change in electronic structure.

A pronounced change in RIXS spectra was found in the high-pressure dimerized phase above $4 \mathrm{GPa}$. The comparison of spectra at ambient and high-pressure phases is displayed in Fig. 2(b). The $0.7 \mathrm{eV}$ peak is suppressed almost completely. The one at $3.5 \mathrm{eV}$ is significantly broadened but remains in the high-pressure phase. Instead of the $0.7 \mathrm{eV}$ peak, a broad continuum spreads roughly from 0.5 to $2.0 \mathrm{eV}$, and a shoulderlike feature around $2.8 \mathrm{eV}$ emerges. The drastic change in RIXS spectra, along with the suppression of the $0.7 \mathrm{eV}$ peak, clearly points to the collapse of the spin-orbit coupling splitting of $J_{\text {eff }}=1 / 2$ and $J_{\text {eff }}=3 / 2$ states in the high-pressure phase. We note that the spectrum of the low pressure phase was recovered after depressurization, indicating that the irreversible chemical change in the sample due to the $\mathrm{X}$-ray irradiation and/or the pressure is not the prime cause of the change in RIXS spectra.

\section{Formation of the molecular orbital in the $\mathrm{Ir}_{2}$ dimers}

The electronic structure calculations were performed using the refined structural parameters in the ambient (Table S1) and high-pressure (Table I) phases, as shown in the plots of partial densities of states from the relevant orbitals in Figs. 3(a) and 3 (b), respectively. Spin-orbit coupling was incorporated into the calculation. On-site Coulomb $U$ was not explicitly introduced, which makes the ambient pressure phase metallic. In Fig. 3(a) for the ambient pressure phase, the splitting of $t_{2 g}$-derived bands into the $\left(J_{\text {eff }}=3 / 2\right)$-derived bands around $-1 \mathrm{eV}$ and $\left(J_{\text {eff }}=1 / 2\right)$-derived bands around the Fermi energy by spin-orbit coupling is clearly seen as in the previous calculations $[19,36]$. Small but appreciable mixing between $J_{\text {eff }}=3 / 2$ and $1 / 2$ can be recognized as in other Ir oxides due to the presence of trigonal distortion and/or the $J_{\text {eff }}=1 / 2 \leftrightarrow$ $3 / 2$ hopping between the neighboring Ir sites [53,54].

In stark contrast, the $d_{x y}, d_{y z}$, and $d_{z x}$ orbitals, instead of the spin-orbital-entangled $J_{\text {eff }}=1 / 2$ and $3 / 2$ states, appear to represent the character of the bands in the high-pressure monoclinic phase in Fig. 3(b). The $d_{z x}$ orbital, directed along the dimer bond ( $\mathrm{Y}$ bond), provides the dominant character of the two subbands stemming from $t_{2 g}$-derived bands: the lowest $(-1.7 \mathrm{eV})$ occupied subband and the highest $(+0.7 \mathrm{eV})$ empty subband. The two subbands with predominant $d_{z x}$ character can be assigned to the bonding and the antibonding states of $\mathrm{Ir}_{2}$ dimer molecules with a splitting energy of $\sim 2.4 \mathrm{eV}$. The other $t_{2 g}$ orbitals, $d_{x y}$ and $d_{y z}$ orbitals, mainly contribute to the subbands between the $d_{z x}$-bonding and -antibonding subbands. Because of the degeneracy of $d_{x y}$ and $d_{y z}$ orbitals, strong spin-orbital entanglement is expected for these $d_{x y}$ - and $d_{y z}$-derived subbands, which are denoted as entangled $x y-y z$ orbitals with different colors in Fig. 3(b). Since the hybridization of entangled $x y-y z$ orbitals between the nearest-neighbor Ir atoms is much weaker than that of $d_{z x}$ orbitals, it is natural that they reside in between the bonding and antibonding orbitals of $d_{z x}$. As a result, $\operatorname{Ir} d^{5}$ electrons fill up the bonding $d_{z x}$ subbands and the four entangled $x y-y z$ subbands. An energy gap is formed between the entangled $x y-y z$ subbands and the empty antibonding $d_{z x}$ subband, yielding a band-insulating state. The bandwidth of occupied states increases appreciably compared with that at ambient pressure, which is consistent with the broad feature observed in the RIXS spectra at the low-energy region up to $\sim 2.0 \mathrm{eV}$. We note that the effect of electron correlations may narrow the bandwidth and make the RIXS peaks sharper. The $e_{g}$ orbitals are almost degenerate at ambient pressure but split appreciably in the high-pressure phase due to the strong distortion of $\mathrm{IrO}_{6}$ octahedra. This accounts for the broadening of the RIXS peak at $3.5 \mathrm{eV}$ in the high-pressure phase.

The formation of $\operatorname{Ir}_{2}$ dimers in the hyperhoneycomb lattice gives rise to bonding and antibonding molecular-orbital states made of $d_{z x}$ orbitals in the bonding plane. The large bondingantibonding splitting stabilizes a $d_{z x}$-orbital-dominant antibonding state of $t_{2 g}$ holes and makes the system a band insulator, which is consistent with the negligible XMCD above $4 \mathrm{GPa}[15,36]$. The emergence of the $d_{z x}$-orbital-dominant state results in the collapse of the $J_{\text {eff }}=1 / 2$ state.

\section{DISCUSSION}

The present result points to a competition between the spinorbital entanglement and the dimerization in $\beta-\mathrm{Li}_{2} \mathrm{IrO}_{3}$. The former mixes up the different orbital states to create the orbital moment. The latter selects a specific orbital to gain bonding energy. It was theoretically discussed that $\beta-\mathrm{Li}_{2} \mathrm{IrO}_{3}$ shows an intrinsic instability towards the formation of $\operatorname{Ir}_{2}$ dimers when spin-orbit coupling is neglected [34]. At ambient pressure, the spin-orbit coupling overcomes the dimer instability, and the $J_{\text {eff }}=1 / 2$ state is formed. By increasing the overlap of orbitals, the instability is enhanced, and eventually, the dimer phase shows up. Similar dimerization under pressure was recently identified in another honeycomb iridate, $\alpha-\mathrm{Li}_{2} \mathrm{IrO}_{3}$ [52,55], and also the $4 d$ ruthenium chloride $\alpha-\mathrm{RuCl}_{3}[56,57]$. We note that in the previous theoretical approaches, the dimerization is predicted to take place within the $\mathrm{Z}$ bond [34]. In all $\alpha-\mathrm{Li}_{2} \mathrm{IrO}_{3}, \alpha-\mathrm{RuCl}_{3}$, and $\beta-\mathrm{Li}_{2} \mathrm{IrO}_{3}$, the dimers are formed in the zigzag chains made of the $\mathrm{X}$ and $\mathrm{Y}$ bonds rather than the $\mathrm{Z}$ bond. The anisotropic lattice contraction of the zigzag chains under pressure seems to be closely related to the preferable dimerization in the zigzag chains, although its origin remains to be settled. The dimerization of transitionmetal ions has been frequently seen not only in honeycombbased $5 d^{5}$ iridates but also in a wide variety of honeycombbased $3 d$ and $4 d$ oxides and halides, including $\alpha-\mathrm{TiCl}_{3}\left(3 d^{1}\right)$ [58], $\alpha-\mathrm{MoCl}_{3}\left(4 d^{3}\right)$ [59], and $\mathrm{Li}_{2} \mathrm{RuO}_{3}\left(4 d^{4}\right)$ [60], even at ambient pressure. The occurrence of dimerization only under a high pressure may reflect that the competition with the spin-orbital-entangled phase is much more significant in the $5 d^{5}$ iridates with spin-orbit coupling of $\sim 0.5 \mathrm{eV}$, much larger than those of the ambient pressure dimerized compounds. For $\alpha-\mathrm{RuCl}_{3}$, despite smaller spin-orbit coupling of $\sim 0.1 \mathrm{eV}$, the less distorted crystal structure likely stabilizes the $J_{\text {eff }}=1 / 2$ state at ambient pressure [61].

The dimer transition at $P_{\mathrm{S}} \sim 4 \mathrm{GPa}$ occurs at room temperature in $\beta-\mathrm{Li}_{2} \mathrm{IrO}_{3}$. The disappearance of magnetic ordering in $\beta-\mathrm{Li}_{2} \mathrm{IrO}_{3}$ in the $T=0$ limit was reported to occur at $\sim 2 \mathrm{GPa}$ $[36,38]$. The dimerization at $4 \mathrm{GPa}$ at room temperature could 
be suppressed to a lower pressure with decreasing temperature and may compete with the low-temperature magnetically ordered phase around $2 \mathrm{GPa}$. The low-temperature structure under pressure should be explored to fully disclose the phase competition inherent in the honeycomb-based iridium oxides. In fact, the sister compound $\gamma-\mathrm{Li}_{2} \mathrm{IrO}_{3}$ shows an analogous pressure collapse of the magnetic ordering at $P_{c}=1.5 \mathrm{GPa}$ but no signature of structural dimerization up to $3.3 \mathrm{GPa}$ [37]. It may be interesting to infer that the instability to dimer formation may be relevant for the breakdown of magnetic order in such 3D-based honeycomb iridates.

\section{CONCLUSION}

We studied the crystal and electronic structures of the hyperhoneycomb iridate $\beta-\mathrm{Li}_{2} \mathrm{IrO}_{3}$ in the high-pressure phase above $4 \mathrm{GPa}$. The high-pressure phase is characterized by the formation of $\operatorname{Ir}_{2}$ dimers on the zigzag chains. The spinorbital-entangled $J_{\text {eff }}=1 / 2$ states break down, associated with the stabilization of the bonding state of the neighboring $d_{z x}$ orbitals in the dimer phase. Such competition of spinorbital entanglement and dimer formation are indeed widely observed in honeycomb-based iridates and ruthenium chloride, and we argue it is one of the hallmarks of the physics of these materials.

\section{ACKNOWLEDGMENTS}

We are grateful to D. Haskel, L. S. I. Veiga, S. K. Choi, K. Kitagawa, and R. Dinnebier for helpful discussions. We thank S. K. Choi, H. H. Kim, U. Engelhardt, and K. Syassen for their help in the preparation of DAC experiments. We acknowledge the provision of beam time for the PEARL experiment (Proposal No. RB1710302) to the Science and Technology Facilities Council (STFC) and the allocation of beam time at BL12XU of SPring-8 (Proposals No. 2017-1-1191/2017A4253, No. 2017-1-119-3/2017B4246, and No. 20173-078-3/2018A4260) to the National Synchrotron Radiation Research Center (NSRRC) and the Japan Synchrotron Radiation Research Institute (JASRI). This work was partly supported by the Alexander von Humboldt Foundation, Japan Society for the Promotion of Science (JSPS) KAKENHI (Grants No. JP15H05852, No. JP15K21717, and No. 17H01140), and the JSPS Core-to-Core Program project "Solid-state chemistry for transition-metal oxides."
[1] A. Kitaev, Ann. Phys. (NY) 321, 2 (2006).

[2] J. G. Rau, E. K. H. Lee, and H. Y. Kee, Annu. Rev. Condens. Matter Phys. 7, 195 (2016).

[3] S. M. Winter, A. A. Tsirlin, M. Daghofer, J. van den Brink, Y. Singh, P. Gegenwart, and R. Valentí, J. Phys.: Condens. Matter 29, 493002 (2017).

[4] B. J. Kim, H. Ohsumi, T. Komesu, S. Sakai, T. Morita, H. Takagi, and T. Arima, Science 323, 1329 (2009).

[5] G. Jackeli and G. Khaliullin, Phys. Rev. Lett. 102, 017205 (2009).

[6] Y. Singh and P. Gegenwart, Phys. Rev. B 82, 064412 (2010).

[7] Y. Singh, S. Manni, J. Reuther, T. Berlijn, R. Thomale, W. $\mathrm{Ku}, \mathrm{S}$. Trebst, and P. Gegenwart, Phys. Rev. Lett. 108, 127203 (2012).

[8] X. Liu, T. Berlijn, W.-G. Yin, W. Ku, A. Tsvelik, Y. J. Kim, H. Gretarsson, Y. Singh, P. Gegenwart, and J. P. Hill, Phys. Rev. B 83, 220403(R) (2011).

[9] F. Ye, S. Chi, H. Cao, B. C. Chakoumakos, J. A. FernandezBaca, R. Custelcean, T. F. Qi, O. B. Korneta, and G. Cao, Phys. Rev. B 85, 180403(R) (2012).

[10] S. C. Williams, R. D. Johnson, F. Freund, S. Choi, A. Jesche, I. Kimchi, S. Manni, A. Bombardi, P. Manuel, P. Gegenwart, and R. Coldea, Phys. Rev. B 93, 195158 (2016).

[11] K. W. Plumb, J. P. Clancy, L. J. Sandilands, V. V. Shankar, Y. F. $\mathrm{Hu}, \mathrm{K} . \mathrm{S}$. Burch, H. Y. Kee, and Y. J. Kim, Phys. Rev. B 90, 041112(R) (2014).

[12] R. D. Johnson, S. C. Williams, A. A. Haghighirad, J. Singleton, V. Zapf, P. Manuel, I. I. Mazin, Y. Li, H. O. Jeschke, R. Valentí, and R. Coldea, Phys. Rev. B 92, 235119 (2015).

[13] H. B. Cao, A. Banerjee, J.-Q. Yan, C. A. Bridges, M. D. Lumsden, D. G. Mandrus, D. A. Tennant, B. C. Chakoumakos, and S. E. Nagler, Phys. Rev. B 93, 134423 (2016).

[14] A. Banerjee, C. A. Bridges, J. Q. Yan, A. A. Aczel, L. Li, M. B. Stone, G. E. Granroth, M. D. Lumsden, Y. Yiu,
J. Knolle, S. Bhattacharjee, D. L. Kovrizhin, R. Moessner, D. A. Tennant, D. G. Mandrus, and S. E. Nagler, Nat. Mater. 15, 733 (2016).

[15] T. Takayama, A. Kato, R. Dinnebier, J. Nuss, H. Kono, L. S. I. Veiga, G. Fabbris, D. Haskel, and H. Takagi, Phys. Rev. Lett. 114, 077202 (2015).

[16] K. A. Modic, T. E. Smidt, I. Kimchi, N. P. Breznay, A. Biffin, S. K. Choi, R. D. Johnson, R. Coldea, P. WatkinsCurry, G. T. McCandless, J. Y. Chan, F. Gandara, Z. Islam, A. Vishwanath, A. Shekhter, R. D. McDonald, and J. G. Analytis, Nat. Commun. 5, 4203 (2014).

[17] A. Biffin, R. D. Johnson, S. Choi, F. Freund, S. Manni, A. Bombardi, P. Manuel, P. Gegenwart, and R. Coldea, Phys. Rev. B. 90, 205116 (2014).

[18] A. Biffin, R. D. Johnson, I. Kimchi, R. Morris, A. Bombardi, J. G. Analytis, A. Vishwanath, and R. Coldea, Phys. Rev. Lett. 113, 197201 (2014).

[19] H. S. Kim, E. K. H. Lee, and Y. B. Kim, Europhys. Lett. 112, 67004 (2015).

[20] V. M. Katukuri, R. Yadav, L. Hozoi, S. Nishimoto, and J. van den Brink, Sci. Rep. 6, 29585 (2016).

[21] A. Ruiz, A. Frano, N. P. Breznay, I. Kimchi, T. Helm, I. Oswald, J. Y. Chan, R. J. Birgeneau, Z. Islam, and J. G. Analytis, Nat. Commun. 8, 961 (2017).

[22] V. Todorova, A. Leineweber, L. Kienle, V. Duppel, and M. Jansen, J. Solid State Chem. 184, 1112 (2011).

[23] J. H. Roudebush, K. A. Ross, and R. J. Cava, Dalton Trans. 45, 8783 (2016).

[24] M. Abramchuk, C. Ozsoy-Keskinbora, J. W. Krizan, K. R. Metz, D. C. Bell, and F. Tafti, J. Am. Chem. Soc. 139, 15371 (2017).

[25] K. Kitagawa, T. Takayama, Y. Matsumoto, A. Kato, R. Takano, Y. Kishimoto, S. Bette, R. E. Dinnebier, G. Jackeli, and H. Takagi, Nature (London) 554, 341 (2018). 
[26] S. Bette, T. Takayama, K. Kitagawa, R. Takano, H. Takagi, and R. E. Dinnebier, Dalton Trans. 46, 15216 (2017).

[27] J. A. Sears, Y. Zhao, Z. Xu, J. W. Lynn, and Y. J. Kim, Phys. Rev. B 95, 180411(R) (2017).

[28] R. Hentrich, A. U. B. Wolter, X. Zotos, W. Brenig, D. Nowak, A. Isaeva, T. Doert, A. Banerjee, P. Lampen-Kelley, D. G. Mandrus, S. E. Nagler, J. Sears, Y.-J. Kim, B. Büchner, and C. Hess, Phys. Rev. Lett. 120, 117204 (2018).

[29] J. Zheng, K. Ran, T. Li, J. Wang, P. Wang, B. Liu, Z.-X. Liu, B. Normand, J. Wen, and W. Yu, Phys. Rev. Lett. 119, 227208 (2017).

[30] Y. Kasahara, T. Ohnishi, Y. Mizukami, O. Tanaka, S. Ma, K. Sugii, N. Kurita, H. Tanaka, J. Nasu, Y. Motome, T. Shibauchi, and Y. Matsuda, Nature (London) 559, 227 (2018).

[31] R. Yadav, N. A. Bogdanov, V. M. Katukuri, S. Nishimoto, J. van den Brink, and L. Hozoi, Sci. Rep. 6, 37925 (2016).

[32] K. A. Modic, B. J. Ramshaw, J. B. Betts, N. P. Breznay, J. G. Analytis, R. D. McDonald, and A. Shekhter, Nat. Commun. 8, 180 (2017).

[33] R. Yadav, S. Rachel, L. Hozoi, J. van den Brink, and G. Jackeli, Phys. Rev. B 98, 121107(R) (2018).

[34] H. S. Kim, Y. B. Kim, and H.-Y. Kee, Phys. Rev. B 94, 245127 (2016).

[35] I. Rousochatzakis and N. B. Perkins, Phys. Rev. Lett. 118, 147204 (2017).

[36] L. S. I. Veiga, M. Etter, K. Glazyrin, F. Sun, C. A. Escanhoela, Jr., G. Fabbris, J. R. L. Mardegan, P. S. Malavi, Y. Deng, P. P. Stavropoulos, H.-Y. Kee, W. G. Yang, M. van Veenendaal, J. S. Schilling, T. Takayama, H. Takagi, and D. Haskel, Phys. Rev. B 96, 140402(R) (2017).

[37] N. P. Breznay, A. Ruiz, A. Frano, W. Bi, R. J. Birgeneau, D. Haskel, and J. G. Analytis, Phys. Rev. B 96, 020402(R) (2017).

[38] M. Majumder, R. S. Manna, G. Simutis, J. C. Orain, T. Dey, F. Freund, A. Jesche, R. Khasanov, P. K. Biswas, E. Bykova, N. Dubrovinskaia, L. S. Dubrovinsky, R. Yadav, L. Hozoi, S. Nishimoto, A. A. Tsirlin, and P. Gegenwart, Phys. Rev. Lett. 120, 237202 (2018).

[39] C. L. Bull, N. P. Funnell, M. G. Tucker, S. Hull, D. J. Francis, and W. G. Marshall, High Pressure Res 36, 493 (2016).

[40] J. M. Besson, R. J. Nelmes, G. Hamel, J. S. Loveday, G. Weill, and S. Hull, Physica B (Amsterdam, Neth.) 180-181, 907 (1992).

[41] W. G. Marshall and D. J. Francis, J. Appl. Crystallogr. 35, 122 (2002).

[42] A. C. Hannon, A. S. Gibbs, and H. Takagi, J. Appl. Crystallogr. 51, 854 (2018).

[43] B. H. Toby, J. Appl. Crystallogr. 34, 210 (2001).

[44] See Supplemental Material, which includes Ref. [62-64], at http://link.aps.org/supplemental/10.1103/PhysRevB.99.125127 for the experimental details of neutron diffraction and RIXS measurements.

[45] H. Gretarsson, J. P. Clancy, X. Liu, J. P. Hill, E. Bozin, Y. Singh, S. Manni, P. Gegenwart, J. Kim, A. H. Said, D. Casa, T. Gog, M. H. Upton, H. S. Kim, J. Yu, V. M. Katukuri, L. Hozoi, J. van den Brink, and Y.-J. Kim, Phys. Rev. Lett. 110, 076402 (2013).
[46] V. Antonov, B. Harmon, and A. Yaresko, Electronic Structure and Magneto-Optical Properties of Solids (Kluwer Academic, Dordrecht, 2004).

[47] K. Momma and F. Izumi, J. Appl. Crystallogr. 44, 1272 (2011).

[48] S. V. Streltsov and D. I. Khomskii, Phys. Usp. 60, 1121 (2017).

[49] K. Ishii, I. Jarrige, M. Yoshida, K. Ikeuchi, J. Mizuki, K. Ohashi, T. Takayama, J. Matsuno, and H. Takagi, Phys. Rev. B 83, 115121 (2011).

[50] J. Kim, D. Casa, M. H. Upton, T. Gog, Y.-J. Kim, J. F. Mitchell, M. van Veenendaal, M. Daghofer, J. van den Brink, G. Khaliullin, and B. J. Kim, Phys. Rev. Lett. 108, 177003 (2012).

[51] X. Liu, V. M. Katukuri, L. Hozoi, W. G. Yin, M. P. M. Dean, M. H. Upton, J. Kim, D. Casa, A. Said, T. Gog, T. F. Qi, G. Cao, A. M. Tsvelik, J. van den Brink, and J. P. Hill, Phys. Rev. Lett. 109, 157401 (2012).

[52] J. P. Clancy, H. Gretarsson, J. A. Sears, Y. Singh, S. Desgreniers, K. Mehlawat, S. Layek, G. Kh. Rozenberg, Y. Ding, M. H. Upton, D. Casa, N. Chen, J. Im, Y. Lee, R. Yadav, L. Hozoi, D. Efremov, J. van den Brink, and Y.-J. Kim, npj Quantum Mater. 3, 35 (2018).

[53] H. Shinaoka, S. Hoshino, M. Troyer, and P. Werner, Phys. Rev. Lett. 115, 156401 (2015).

[54] C. H. Sohn, H. S. Kim, T. F. Qi, D. W. Jeong, H. J. Park, H. K. Yoo, H. H. Kim, J. Y. Kim, T. D. Kang, D. Y. Cho, G. Cao, J. Yu, S. J. Moon, and T. W. Noh, Phys. Rev. B 88, 085125 (2013).

[55] V. Hermann, M. Altmeyer, J. Ebad-Allah, F. Freund, A. Jesche, A. A. Tsirlin, M. Hanfland, P. Gegenwart, I. I. Mazin, D. I. Khomskii, R. Valentí, and C. A. Kuntscher, Phys. Rev. B 97, 020104(R) (2018).

[56] G. Bastien, G. Garbarino, R. Yadav, F. J. Martinez-Casado, R. B. Rodríguez, Q. Stahl, M. Kusch, S. P. Limandri, R. Ray, P. Lampen-Kelley, D. G. Mandrus, S. E. Nagler, M. Roslova, A. Isaeva, T. Doert, L. Hozoi, A. U. B. Wolter, B. Büchner, J. Geck, and J. van den Brink, Phys. Rev. B 97, 241108(R) (2018).

[57] T. Biesner, S. Biswas, W. Li, Y. Saito, A. Pustogow, M. Altmeyer, A. U. B. Wolter, B. Büchner, M. Roslova, T. Doert, S. M. Winter, R. Valentí, and M. Dressel, Phys. Rev. B 97, 220401(R) (2018).

[58] S. Ogawa, J. Phys. Soc. Jpn. 15, 1901 (1960).

[59] M. A. McGuire, J. Yan, P. Lampen-Kelley, A. F. May, V. R. Cooper, L. Lindsay, A. Puretzky, L. Liang, S. KC, E. Cakmak, S. Calder, and B. C. Sales, Phys. Rev. Mater. 1, 064001 (2017).

[60] Y. Miura, Y. Yasui, M. Sato, N. Igawa, and K. Kakurai, J. Phys. Soc. Jpn. 76, 033705 (2007).

[61] S. Agrestini, C.-Y. Kuo, K.-T. Ko, Z. Hu, D. Kasinathan, H. B. Vasili, J. Herrero-Martin, S. M. Valvidares, E. Pellegrin, L.-Y. Jang, A. Henschel, M. Schmidt, A. Tanaka, and L. H. Tjeng, Phys. Rev. B 96, 161107(R) (2017).

[62] O. Arnold et al., Nucl. Instrum. Methods Phys. Res., Sect. A 764, 156 (2014).

[63] N. Tateiwa and Y. Haga, Rev. Sci. Instrum. 80, 123901 (2009).

[64] K. Takemura, J. Appl. Phys. 89, 662 (2001) 


\title{
Supplemental Materials for \\ "Pressure-induced collapse of spin-orbital Mott state in the hyperhoneycomb iridate $\beta$ - $\mathrm{Li}_{2} \mathrm{IrO}_{3}$ "
}

\author{
T. Takayama ${ }^{1,2}$, A. Krajewska ${ }^{1,2}$, A. S. Gibbs ${ }^{3}$, A. N. Yaresko ${ }^{1}$, H. Ishii ${ }^{4}$, H. Yamaoka ${ }^{5}$, K. Ishii ${ }^{6}$, N. \\ Hiraoka $^{4}$, N. P. Funnell ${ }^{3}$, C. L. Bull ${ }^{3}$ and H. Takagi ${ }^{1,2,7}$ \\ ${ }^{1}$ Max Planck Institute for Solid State Research, Heisenbergstrasse 1, 70569 Stuttgart, Germany. \\ ${ }^{2}$ Insitute for Functional Matter and Quantum Technologies, University of Stuttgart, Pfaffenwaldring \\ 57, 70550 Stuttgart, Germany. \\ ${ }^{3}$ ISIS Facility, STFC Rutherford Appleton Laboratory, Chilton, Didcot, Oxon OX11 0QX, UK. \\ ${ }^{4}$ National Synchrotron Radiation Research Center, Hsinchu 30076, Taiwan. \\ ${ }^{5}$ RIKEN SPring-8 Center, Sayo, Hyogo 679-5148, Japan. \\ ${ }^{6}$ Synchrotron Radiation Research Center, National Institutes for Quantum and Radiological Science \\ and Technology, Sayo, Hyogo 679-5148, Japan. \\ ${ }^{7}$ Department of Physics, University of Tokyo, 7-3-1 Hongo, Bunkyo-ku, Tokyo 113-0033, Japan.
}

\section{Structural refinements with neutron diffraction}

The powder neutron diffraction data were collected at the Pearl beamline of the ISIS neutron source. The time-of-flight data were normalized and corrected using in-house software [62]. A beam-line-developed correction for the wavelength and scattering-angle dependence of the neutron attenuation by the ZTA anvil and Ti-Zr gasket materials was applied to the observed pattern [39]. The results of Rietveld refinement at ambient pressure, 2.6(1) and 4.4(1) GPa are shown in Figs. $\mathrm{S} 1$ (a), (b) and $\mathrm{S} 2$, respectively. In Fig. S1, the crystal structure of $\beta-\mathrm{Li}_{2} \mathrm{IrO}_{3}$ is refined to be orthorhombic as reported before. The refined structural parameters are listed in Table S1 and S2. For the high-pressure phase in Fig. S2, the refinement was performed assuming the monoclinic cell with the space group of $C 2 / c$ (No. 15). The refined crystal structure is illustrated in Fig. S3(a), and the Ir-O bond lengths are shown in Fig. S3(b). The Ir-O lengths are similar to those of the ambient pressure phase, and the Ir-Ir lengths are mostly controlled by the Ir-O-Ir angles.

The comparison of the refined structures by single crystal XRD (Ref. [36]) and the neutron diffractions is shown in Table S3. Although there are small discrepancies, for example, the Ir-Ir lengths in the ambient pressure phase and Ir-O lengths in the high-pressure phase, the refined structures by the two different probes are found to be essentially consistent. 

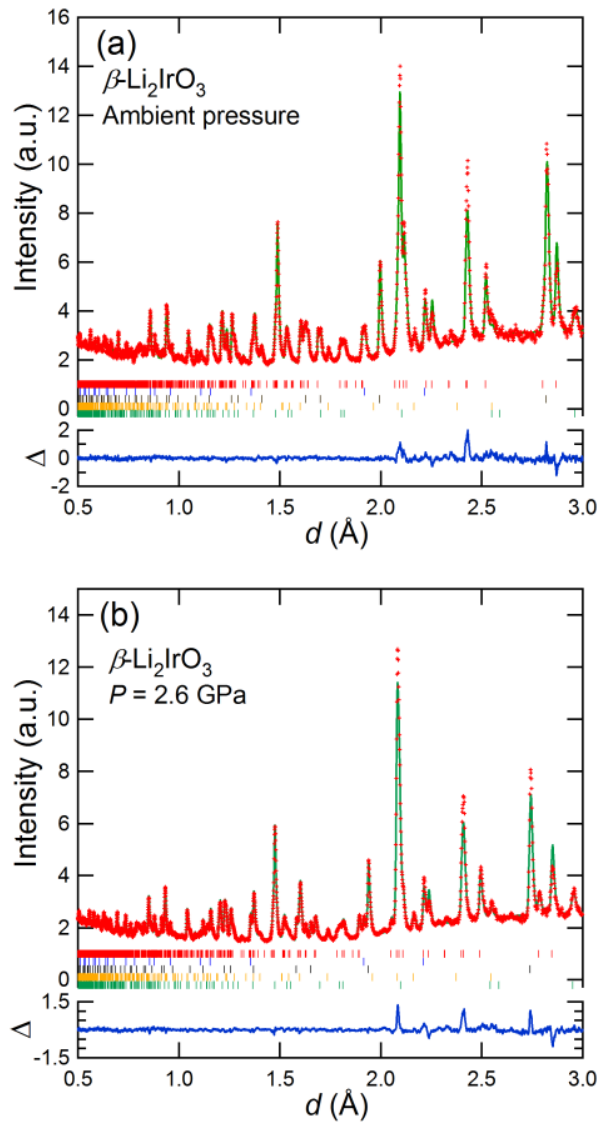

Fig. S1. Powder neutron diffraction pattern and Rietveld analysis of $\beta-{ }^{7} \mathrm{Li}_{2}{ }^{193} \mathrm{IrO}_{3}$ polycrystalline sample at room temperature, collected at (a) ambient pressure and (b) 2.6(1) GPa. The red dots and green line represent the observed pattern and the Rietveld fit profile respectively. The red, blue, black, yellow and green bars indicate the reflection positions of $\beta$ - $\mathrm{Li}_{2} \mathrm{IrO}_{3}$, metallic $\mathrm{Ir}, \mathrm{NaCl}, \mathrm{Al}_{2} \mathrm{O}_{3}$ and $\mathrm{ZrO}_{2}$, respectively. The blue line shows the difference between the observed and calculated profiles $\Delta$. The crystal structure of $\beta-\mathrm{Li}_{2} \mathrm{IrO}_{3}$ is refined as an orthorhombic structure (Space group: Fddd, No.70). 
Table S1. Refined structural parameters of $\beta-\mathrm{Li}_{2} \mathrm{IrO}_{3}$ at ambient pressure based on neutron diffraction. The space group is $F d d d$ (No. 70) and $Z=16$. The lattice parameters are $a=5.9055(4)$

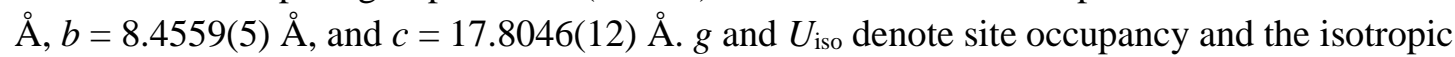
displacement parameter, respectively. $U_{\text {iso }}$ was constrained to be equal across sites containing the same element during the refinement. The refinement indices are $R_{p}=3.32 \%, R_{w p}=3.96 \%$, and $\chi^{2}$ $=1.236$.

\begin{tabular}{|c|c|c|c|c|c|c|}
\hline Atom & site & $g$ & $x$ & $y$ & $z$ & $U_{\text {iso }}\left(\AA^{2}\right)$ \\
\hline Li1 & $16 g$ & 1 & $1 / 8$ & $1 / 8$ & $0.0574(7)$ & $0.0101(15)$ \\
\hline Li2 & $16 g$ & 1 & $1 / 8$ & $1 / 8$ & $0.8719(8)$ & $0.0101(15)$ \\
\hline Ir1 & $16 g$ & 1 & $1 / 8$ & $1 / 8$ & $0.7063(3)$ & $0.0091(5)$ \\
\hline O1 & $16 e$ & 1 & $0.8607(10)$ & $1 / 8$ & $1 / 8$ & $0.0075(3)$ \\
\hline O2 & $32 h$ & 1 & $0.6363(10)$ & $0.3662(4)$ & $0.0381(3)$ & $0.0075(3)$ \\
\hline
\end{tabular}

Table S2. Refined structural parameters of $\beta-\mathrm{Li}_{2} \mathrm{IrO}_{3}$ under a pressure of 2.6(1) GPa based on neutron diffraction. The space group is $F d d d$ (No. 70) and $Z=16$. The lattice parameters are $a=$ 5.8641(4) $\AA, b=8.3604(5) \AA$, and $c=17.687(1) \AA . g$ and $U_{\text {iso }}$ denote site occupancy and the isotropic displacement parameter, respectively. $U_{\text {iso }}$ was constrained to be equal across sites containing the same element during the refinement. The refinement indices are $R_{p}=3.46 \%, R_{w p}=$ $2.92 \%$ and $\chi^{2}=2.286$.

\begin{tabular}{|c|c|c|c|c|c|c|}
\hline Atom & site & $g$ & $x$ & $y$ & $z$ & $U_{\text {iso }}\left(\AA^{2}\right)$ \\
\hline Li1 & $16 g$ & 1 & $1 / 8$ & $1 / 8$ & $0.0597(7)$ & $0.0138(16)$ \\
\hline Li2 & $16 g$ & 1 & $1 / 8$ & $1 / 8$ & $0.8725(9)$ & $0.0138(16)$ \\
\hline Ir1 & $16 g$ & 1 & $1 / 8$ & $1 / 8$ & $0.7076(3)$ & $0.0103(4)$ \\
\hline O1 & $16 e$ & 1 & $0.8595(13)$ & $1 / 8$ & $1 / 8$ & $0.0076(3)$ \\
\hline O2 & $32 h$ & 1 & $0.6341(13)$ & $0.3667(4)$ & $0.0395(3)$ & $0.0076(3)$ \\
\hline
\end{tabular}




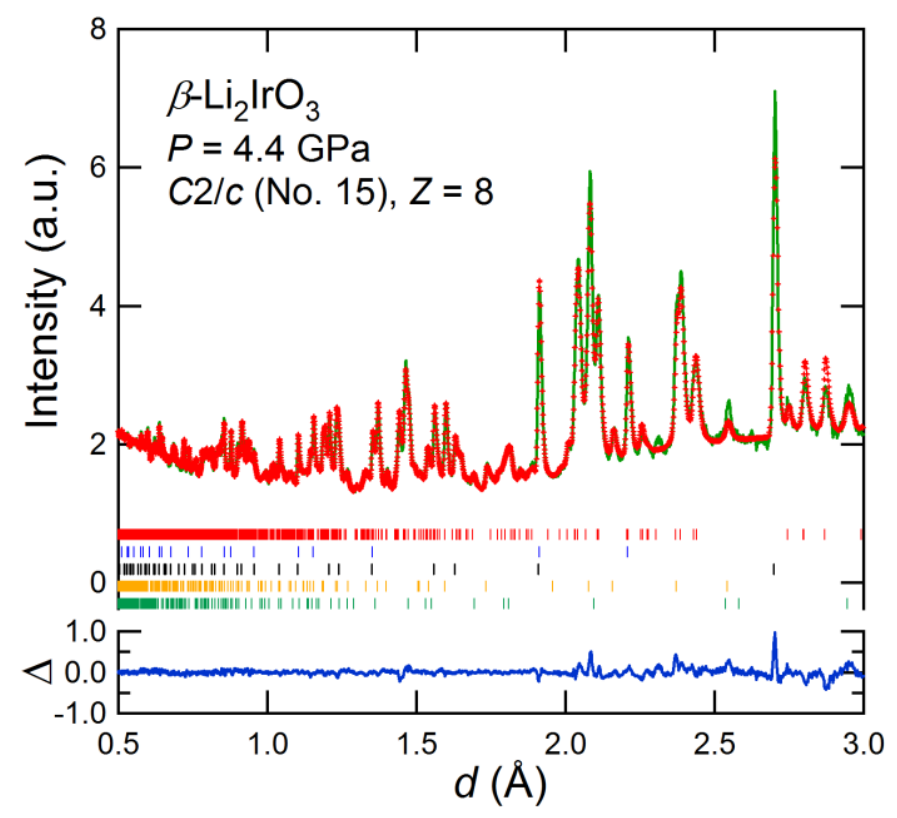

Fig. S2. Powder neutron diffraction pattern and Rietveld analysis of $\beta-{ }^{7} \mathrm{Li}_{2}{ }^{193} \mathrm{IrO}_{3}$ polycrystalline sample in the high-pressure phase at 4.4(1) GPa. The red dots and green line represent the observed pattern and the Rietveld fit profile respectively. The red, blue, black, yellow and green bars indicate the reflection positions of $\beta$ - $\mathrm{Li}_{2} \mathrm{IrO}_{3}$, metallic Ir, $\mathrm{NaCl}, \mathrm{Al}_{2} \mathrm{O}_{3}$ and $\mathrm{ZrO}_{2}$, respectively. The blue line shows the difference between the observed and calculated profiles $\Delta$. The crystal structure of $\beta$ - $\mathrm{Li}_{2} \mathrm{IrO}_{3}$ is refined as a monoclinic structure (Space group: $C 2 / c$, No.15). The result of refinement is listed in Table I of main text.

(a)

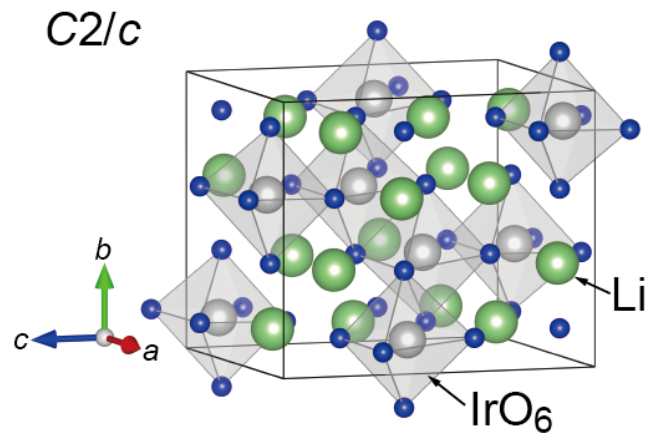

(b)

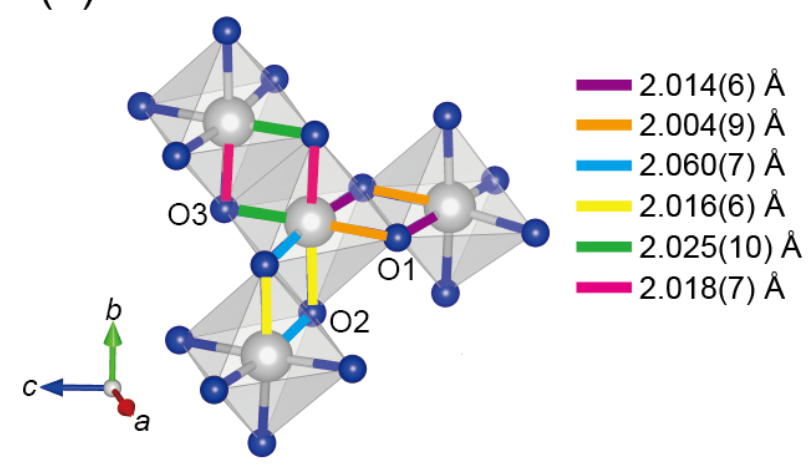

Fig. S3. (a) Crystal structure of $\beta$ - $\mathrm{Li}_{2} \mathrm{IrO}_{3}$ in the high-pressure monoclinic phase. (b) The local structure around an $\mathrm{IrO}_{6}$ octahedra displaying the $\mathrm{Ir}-\mathrm{O}$ bond lengths. 
Table S3. Comparison of refined structures obtained by powder neutron diffraction (this work) and by the single crystal x-ray diffraction (Ref. [36]). "ap" and "ip" in the Ir-O bond lengths represent apical (along the $b$-axis) and in-plane (in the $a c$-plane) Ir-O lengths, respectively.

\section{Powder neutron diffraction (This work)}

\begin{tabular}{|l|l|l|l|}
\hline Pressure $(\mathrm{GPa})$ & Ir-O distance $(\AA)$ & Ir-Ir distance $(\AA)$ & Ir-O-Ir angle $\left(^{\mathrm{o}}\right)$ \\
\hline Ambient pressure & $2.015(6)(\mathrm{Ir}-\mathrm{O} 1)$ & $3.002(4)(\mathrm{X}, \mathrm{Y})$ & $92.6(3)(\mathrm{Ir}-\mathrm{O} 1-\mathrm{Ir})$ \\
& $2.043(4)(\mathrm{Ir}-\mathrm{O} 2 \mathrm{ap})$ & $2.913(8)(\mathrm{Z})$ & $95.2(3)(\mathrm{Ir}-\mathrm{O} 2-\mathrm{Ir})$ \\
& $2.022(7)(\mathrm{Ir}-\mathrm{O} 2 \mathrm{ip})$ & & \\
\hline $2.6(1)$ & $2.006(7)(\mathrm{Ir}-\mathrm{O} 1)$ & $2.961(4)(\mathrm{X}, \mathrm{Y})$ & $93.5(3)(\mathrm{Ir}-\mathrm{O} 1-\mathrm{Ir})$ \\
& $2.022(4)(\mathrm{Ir}-\mathrm{O} 2 \mathrm{ap})$ & $2.922(8)(\mathrm{Z})$ & $94.1(3)(\mathrm{Ir}-\mathrm{O} 2-\mathrm{Ir})$ \\
& $2.025(8)(\mathrm{Ir}-\mathrm{O} 2 \mathrm{ip})$ & & \\
\hline $4.4(1)$ & $2.014(6)(\mathrm{Ir}-\mathrm{O} 1)$ & $3.069(5)(\mathrm{X})$ & $97.6(3)(\mathrm{Ir}-\mathrm{O} 1-\mathrm{Ir})$ \\
& $2.004(9)(\mathrm{Ir}-\mathrm{O} 1)$ & $2.663(5)(\mathrm{Y})$ & $97.6(3)(\mathrm{Ir}-\mathrm{O} 2-\mathrm{Ir})$ \\
& $2.060(7)(\mathrm{Ir}-\mathrm{O} 2)$ & $3.026(6)(\mathrm{Z})$ & $82.5(4)(\mathrm{Ir}-\mathrm{O} 3-\mathrm{Ir})$ \\
& $2.016(6)(\mathrm{Ir}-\mathrm{O} 2)$ & & \\
& $2.025(10)(\mathrm{Ir}-\mathrm{O} 3)$ & & \\
& $2.018(7)(\mathrm{Ir}-\mathrm{O} 3)$ & & \\
\hline
\end{tabular}

Single crystal x-ray diffraction (from Ref. [36])

\begin{tabular}{|c|c|c|c|}
\hline Pressure $(\mathrm{GPa})$ & Ir-O distance $(\AA)$ & Ir-Ir distance $(\AA)$ & Ir-O-Ir angle $\left({ }^{\circ}\right)$ \\
\hline 0.25 & $\begin{array}{l}2.013(6)(\mathrm{Ir}-\mathrm{O} 1) \\
2.015(4)(\mathrm{Ir}-\mathrm{O} 2 \mathrm{ap}) \\
2.004(6)(\mathrm{Ir}-\mathrm{O} 2 \mathrm{ip})\end{array}$ & $\begin{array}{l}2.9589(3)(\mathrm{X}, \mathrm{Y}) \\
2.9528(5)(\mathrm{Z})\end{array}$ & $\begin{array}{l}\text { 94.4(3) (Ir-O1-Ir) } \\
\text { 94.82(18) (Ir-O2-Ir) }\end{array}$ \\
\hline 0.93 & $\begin{array}{l}2.014(6)(\mathrm{Ir}-\mathrm{O} 1) \\
2.012(5)(\mathrm{Ir}-\mathrm{O} 2 \mathrm{ap}) \\
2.001(6)(\mathrm{Ir}-\mathrm{O} 2 \mathrm{ip})\end{array}$ & $\begin{array}{l}2.9499(6)(\mathrm{X}, \mathrm{Y}) \\
2.9499(4)(\mathrm{Z})\end{array}$ & $\begin{array}{l}94.2(3)(\mathrm{Ir}-\mathrm{O} 1-\mathrm{Ir}) \\
94.62(19)(\mathrm{Ir}-\mathrm{O} 2-\mathrm{Ir})\end{array}$ \\
\hline 1.73 & $\begin{array}{l}2.019(9)(\mathrm{Ir}-\mathrm{O} 1) \\
2.006(7)(\mathrm{Ir}-\mathrm{O} 2 \mathrm{ap}) \\
1.986(9)(\mathrm{Ir}-\mathrm{O} 2 \mathrm{ip})\end{array}$ & $\begin{array}{l}2.9422(8)(\mathrm{X}, \mathrm{Y}) \\
2.9458(15)(\mathrm{Z})\end{array}$ & $\begin{array}{l}\text { 93.7(5) (Ir-O1-Ir) } \\
95.0(4)(\mathrm{Ir}-\mathrm{O} 2-\mathrm{Ir})\end{array}$ \\
\hline 3.08 & $\begin{array}{l}2.007(5)(\mathrm{Ir}-\mathrm{O} 1) \\
2.002(5)(\mathrm{Ir}-\mathrm{O} 2 \mathrm{ap}) \\
2.000(4)(\mathrm{Ir}-\mathrm{O} 2 \mathrm{ip})\end{array}$ & $\begin{array}{l}2.9246(7)(\mathrm{X}, \mathrm{Y}) \\
2.9379(16)(\mathrm{Z})\end{array}$ & $\begin{array}{l}\text { 94.1(3) (Ir-O1-Ir) } \\
\text { 93.89(16) (Ir-O2-Ir) }\end{array}$ \\
\hline 5.8 & $\begin{array}{l}2.042(8)(\mathrm{Ir}-\mathrm{O} 1) \\
1.999(11)(\mathrm{Ir}-\mathrm{O} 1) \\
1.977(13)(\mathrm{Ir}-\mathrm{O} 2) \\
2.019(8)(\mathrm{Ir}-\mathrm{O} 2) \\
1.972(12)(\mathrm{Ir}-\mathrm{O} 3) \\
1.995(15)(\mathrm{Ir}-\mathrm{O} 3)\end{array}$ & $\begin{array}{l}3.0143(13)(\mathrm{X}) \\
2.6609(14)(\mathrm{Y}) \\
3.0129(15)(\mathrm{Z})\end{array}$ & $\begin{array}{l}\text { 96.5(5) (Ir-O1-Ir) } \\
98.3(5)(\mathrm{Ir}-\mathrm{O} 2-\mathrm{Ir}) \\
\text { 84.3(6) (Ir-O3-Ir) }\end{array}$ \\
\hline
\end{tabular}




\section{Lattice constants of $\beta$ - $\mathrm{Li}_{2} \mathrm{IrO}_{3}$ under pressure}

The evolution of lattice constants by pressure, refined by neutron diffraction at room temperature, is shown in Fig. S3. To compare the lattice constants between the orthorhombic structure at ambient pressure and the monoclinic structure above the critical pressure $P_{\mathrm{S}}$, the orthorhombic cell with $F d d d$ space group was converted to the pseudo-monoclinic cell $(C 2 / c)$, and the lattice constants are normalized by the values at ambient pressure.

As reported in Ref. [36], the $b$-axis lattice constant shows a much stronger pressure-dependence than $a$ and $c$. Interestingly, even above $P_{\mathrm{S}}$, namely after the dimerization, it keeps displaying the strong pressure dependence. The origin of this pressure-dependence is worthy of further investigation. We did not observe clear phase-coexistence around $P_{\mathrm{S}}$, and the pressure-induced structural transition takes place rather sharply at $P_{\mathrm{S}} \sim 3.7 \mathrm{GPa}$.

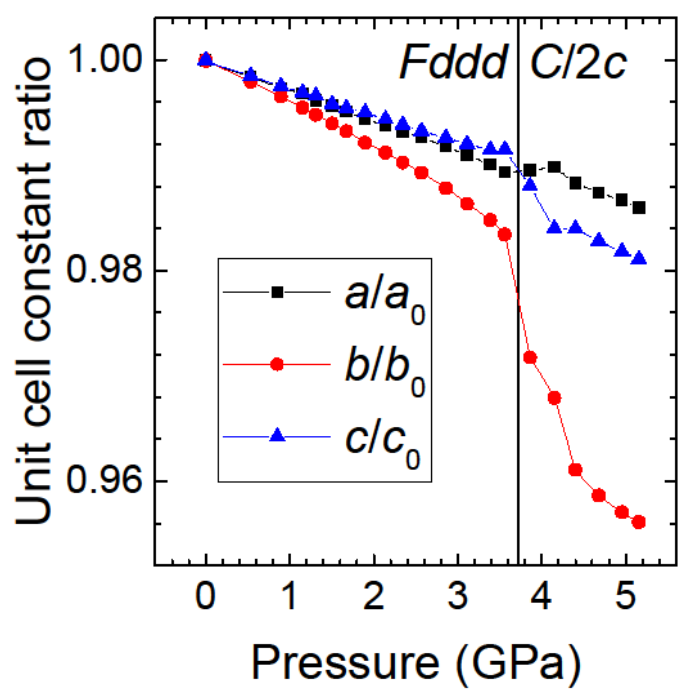

Fig. S4. Pressure-dependent lattice constants of $\beta$ - $\mathrm{Li}_{2} \mathrm{IrO}_{3}$ at room temperature. The lattice constants in the orthorhombic structure are transformed into the ones for the pseudomonoclinic cell $(C 2 / c)$. The lattice constants are normalized by the values at ambient pressure. The vertical line shows the critical pressure $P_{\mathrm{S}} \sim 3.7 \mathrm{GPa}$. 


\section{RIXS spectra in the low-pressure phase}

The change of electronic structures by applying a pressure of 1-2 GPa, within the low-pressure (orthorhombic) phase, was reported for $\beta$ - $\mathrm{Li}_{2} \mathrm{IrO}_{3}$ by x-ray absorption spectra (XAS) [36], and the suppression of magnetic order is seen in the similar pressure range $[36,38]$. To get insights into the electronic structure in the pressure range, the RIXS spectra in the ambient pressure phase are compared in Fig. S5. The spectra are normalized by the intensities of the high-energy tail above 5 $\mathrm{eV}$ as in Fig. 2(b). At both $0.9 \mathrm{GPa}$ and $3.1 \mathrm{GPa}$, the excitation at $\sim 0.7 \mathrm{eV}$ is clearly observed, indicating the presence of $J_{\text {eff }}=1 / 2$ state. The $0.7 \mathrm{eV}$-peak looks a little broadened to the lowenergy side, but it is difficult to pin down this change with the current energy resolution.

Measurements with higher energy resolution and better statistics are required for further exploration for the evolution of electronic structure by pressure in the low-pressure phase. We note that despite the change of branching ratio for XAS under pressure the ratio of the expectation values of spin and orbital moments, $\left\langle L_{z}\right\rangle \mid\left\langle S_{z}\right\rangle$ estimated from x-ray magnetic circular dichroism, remains almost constant up to $3 \mathrm{GPa}$ [36]. This implies that the deviation from $J_{\text {eff }}=1 / 2$ state is not substantial in the low-pressure phase, in contrast to the dimerized high-pressure phase.
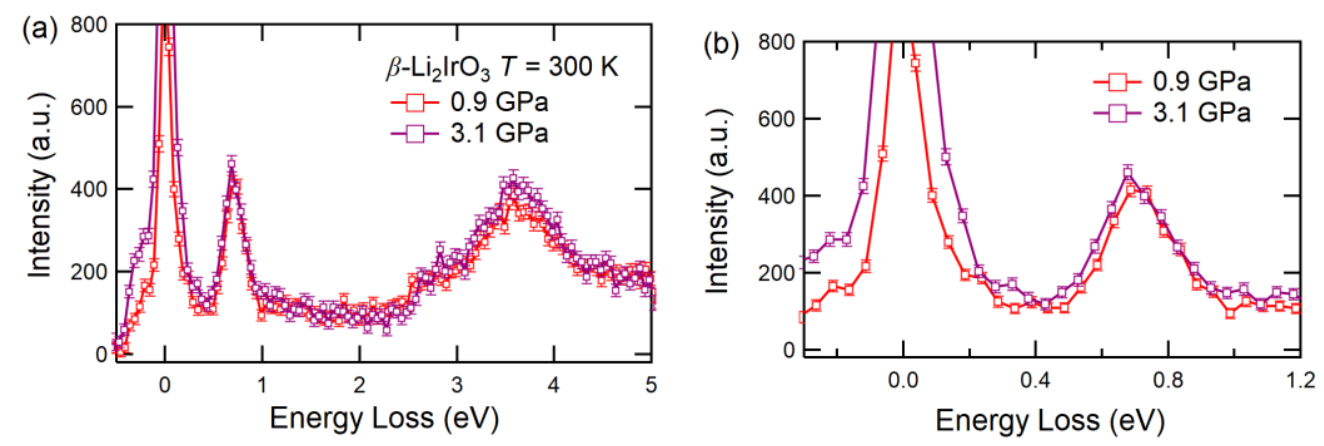

Fig. S5. (a) Ir $L_{3}$-edge RIXS spectra of $\beta$ - $\mathrm{Li}_{2} \mathrm{IrO}_{3}$ in the low-pressure phase at room temperature. The two spectra obtained at 0.9 and $3.1 \mathrm{GPa}$ are displayed. (b) shows the magnified view at a low-energy region. 


\section{Hydrostaticitiy of pressure in the RIXS experiment}

In the RIXS experiment with using a DAC, Fluorinert (1:1 mixture by volume of FC-70 and FC-77) was used as a pressure medium. Since the maximum pressure applied was larger than the hydrostatic limit of Fluorinert [63], we examine the possible influence of pressure-inhomogeneity from the fluorescence spectra of a ruby ball. The representative fluorescence spectra are depicted in Fig. S4(a). From the data, we evaluate the full-width at half-maximum of R1 line and the split of R1 and R2 lines [64] as shown in Fig. S4(b). While the split of lines remained almost same, the FWMH of R1 line increased monotonically up to $7.4 \mathrm{GPa}$, suggesting that the medium solidified at a low pressure around $1 \mathrm{GPa}$ [63]. At high pressures, there may be an effect of non-hydrostaticity to some extent. However, the monotonic increase of non-hydrostaticity cannot account for the drastic change of RIXS spectra across the structural transition (Fig. 2 of main text) and the appearance of new features at high pressures.
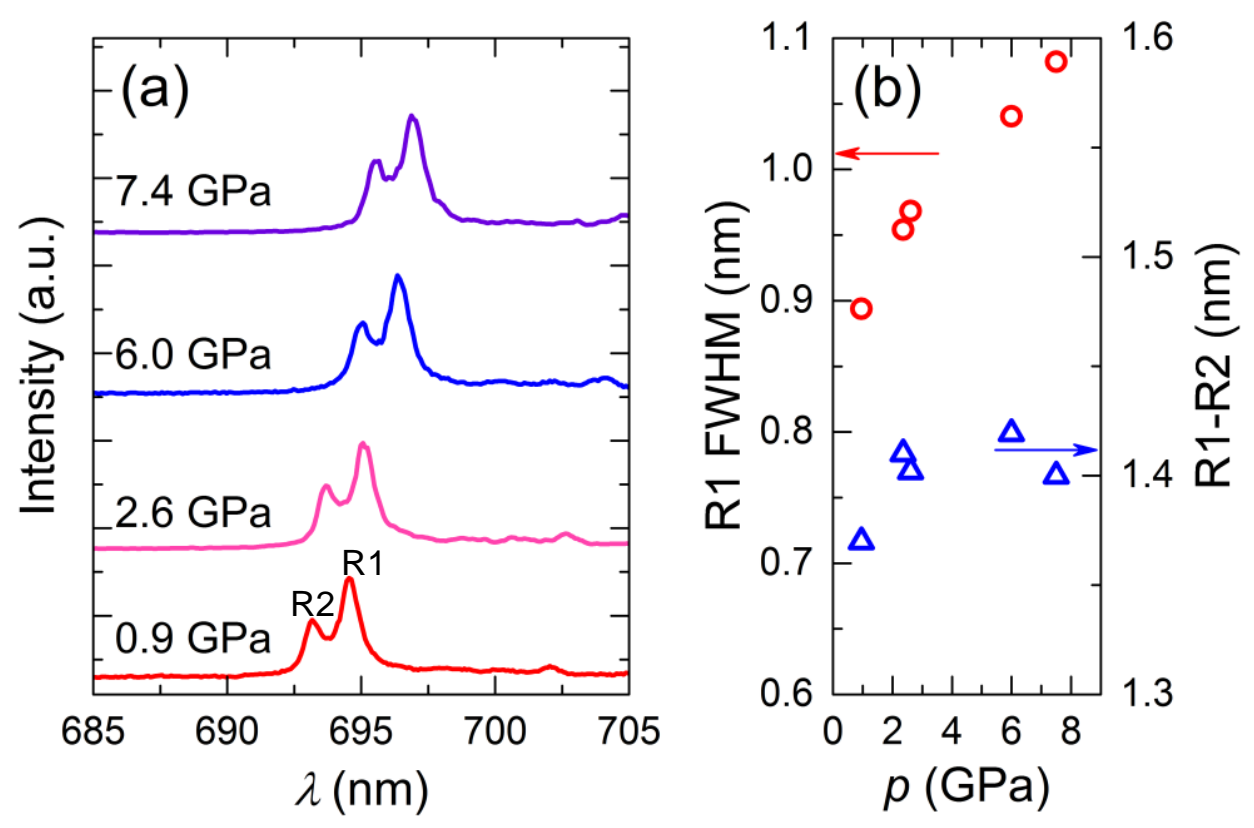

Fig. S6. (a) Fluorescence spectra of a ruby ball introduced in a DAC under pressure. The spectra are displayed with arbitrary offsets. (b) The pressure dependence of full-width at half-maximum (FWHM) of ruby R1 line (red) and the split of R1 and R2 lines (blue). 


\section{References}

[62] O. Arnold et al., Nucl. Instrum. Methods Phys. Res. A 764, 156-166 (2014).

[63] N. Tateiwa and Y. Haga, Rev. Sci. Instrum. 80, 123901 (2009).

[64] K. Takemura, J. Appl. Phys. 89, 662 (2001). 